\title{
Datenschutz und Privatversicherer: Lichtstreifen am Horizont
}

\author{
Hanspeter Kuhn, stv. Generalsekretär FMH \\ Robert Gmür, Rechtsdienst FMH
}

La version française suivra

\footnotetext{
* Die Sozialversicherungsgesetze sehen vor, dass der Versicherer eines haftpflichtigen Dritten Einsichtsrecht in die Akten des Sozialversicherers hat, welcher einen Regressanspruch anmeldet.
}

Korrespondenz:

Rechtsdienst FMH

Postfach 293

CH-3000 Bern

E-Mail:fmhrecht@hin.ch
Veröffentlichungen wie die beiden vorstehenden sind schön und gut, um Positionen zu beziehen und Lösungen aus der einen Sicht vorzuschlagen. Im Bereich der sozialen Krankenversicherung gehen wir davon aus, dass das mit dem Vertrauensarztvertrag geschaffene paritätische Gremium sinnvolle Lösungen wird entwickeln können.

Damit es auch ausserhalb des KVG-Bereichs nicht beim frucht- und folgenlosen Schlagabtausch bleibt, hat sich eine Delegation der FMH Mitte August 2002 mit den Vertretern des SVV an einen Tisch gesetzt. Ein Mitarbeiter des Eidgenössischen Datenschutzbeauftragten war ebenfalls mit von der Partie. Natürlich konnten die grundsätzlichen Fragen erst andiskutiert werden. Für einige Detailfragen wurden aber doch schon konkrete Lösungsansätze formuliert:

1. Zunächst haben die Vertreter des SVV ein zentrales Postulat aus Sicht der FMH anerkannt: Auch für den SVV sei unbestritten, dass die kritische Beurteilung der medizinischen Voraussetzungen der Leistungspflicht der Versicherung/des Versicherers ausschliesslich in die Hand des erfahrenen Versicherungsarztes gehören. Eine erfahrene Krankenschwester in der Versicherungsadministration kann in vielen Fällen durchaus Kostengutsprachen erteilen - eine Ablehnung wegen fehlender Appropriateness (also wegen fehlender «Wirksamkeit, Zweckmässigkeit und Wirtschaftlichkeit») hingegen ist ausschliesslich Sache des beratenden Arztes.

2. Transparenz: Der Versicherer teilt in seiner Korrespondenz mit Ärzten in jedem Fall mit, in welcher Funktion er im vorliegenden Fall tätig ist (z. B. Unfallversicherer gemäss UVG; Taggeldversicherer des Arbeitgebers; Taggeldversicherer des Patienten mit Einzelpolice; Haftpflichtversicherer eines Dritten, gegenüber dem ein Sozialversicherer oder der Patient Schadenersatzansprüche geltend macht).
3. Von den Privatversicherern durchgeführte obligatorische Unfallversicherung gemäss UVG: für den Versicherten heikle Informationen sollen an den beratenden Arzt adressiert werden; der Versicherer sichert zu, dass künftig nirgendwo mehr der Schadenbearbeiter als Sekretär des beratenden Arztes funktioniert. Diese Information wird vielmehr (beispielsweise nach Öffnung des Briefs durch ein Sekretariat der beratenden Ärzte) auf direktem Weg zum für den Fall zuständigen beratenden Arzt gelangen. Als Anwendungshilfe für die Frage, welche Informationen als heikel für den Versicherten gelten, soll eine gemeinsame Liste erarbeitet werden. Offen blieb im August, ob der skizzierte Weg nur für nicht schadenrelevante heikle Informationen zur Verfügung stehen soll (dies die Vorstellung des SVV) oder aber für alle heiklen Informationen.

4. In Regressverfahren bei Haftpflichtfällen sollen Doppelspurigkeiten vermieden werden. Das heisst, der Haftpflichtversicherer konsultiert zunächst die bestehenden Akten des Sozialversicherers (also z.B. die Akten des Unfallversicherers, der die Behandlungskosten und den Lohnausfall nach dem Verkehrsunfall übernommen hat*). Nur wenn tatsächlich noch Fragen offen bleiben, holt der Haftpflichtversicherer einen Bericht vom behandelnden Arzt ein. Dies wird typischerweise für die Abklärung eines geltendgemachten Haushaltsführungsschadens notwendig sein, der ja vom Haftpflichtversicherer umfassender übernommen werden muss, als vom vorleistenden Sozialversicherer. Wenn der Haftpflichtversicherer einen Bericht des beratenden Arztes benötigt, wird er entweder die Fotokopie der entsprechenden Einwilligungserklärung des Patienten beilegen oder das Datum dieser Einwilligungserklärung in seiner Anfrage an den Arzt mitteilen. Welche der beiden Varianten letztlich sinnvoller bzw. einfacher erscheint, ist noch 
abzuklären. Das Datum ist deshalb zentral, weil sich Haftpflichtfälle oft über eine lange Zeit hinziehen und sich der Gesundheitszustand des Patienten seit der früher gegenüber dem Haftpflichtversicherer erteilten Einwilligung in heiklen Bereichen verändert haben kann; beispielsweise kann nach Erteilung der Einwilligung ein Streit über die Frage entstanden sein, ob und wieweit der Patient wieder arbeitsfähig ist.
Diese ersten konkreten Vorschläge sollen in nächster Zeit FMH-intern und bei den Versicherern des SVV auf Praktikabilität und Akzeptanz geprüft werden. Wir sind Ihnen deshalb für Hinweise und Kritik aus der Sicht des praktizierenden und des Spitalarztes dankbar.

An den nächsten Sitzungen sollen die Ergebnisse der internen Konsultationen besprochen werden. 